\title{
CONSTRUCCIONISMO: OBJETOS PARA PENSAR, ENTIDADES PÚBLICAS Y MICROMUNDOS
}

\author{
Eleonora Badilla Saxe ${ }^{1}$ \\ Alejandra Chacón Murillo ${ }^{2}$
}

\begin{abstract}
Resumen: Este artículo presenta una perspectiva pedagógica de la teoría educativa denominada Construccionismo, propuesta por Seymour Papert sobre la base del Constructivismo de Jean Piaget. Papert considera las computadoras como semillas culturales que pueden ejercer una poderosa influencia en el pensamiento de las personas. En particular, se abordan tres de los conceptos clave para el Construccionismo: objetos para pensar, entidades públicas y micromundos
\end{abstract}

Palabras clave: CONSTRUCCIONISMO/ TEORÍA EDUCATIVAI COMPUTADORAS EN EDUCACIÓN/ TECNOLOGÍA DIGITAL/ EDUCACIÓN/ OBJETOS PARA PENSAR/ ENTIDADES PÚBLICAS/ MICROMUNDOS/

Abstract: This article presents a pedagogical perspective of Constuctionism, the educational theory proposed by Seymour Papert, based on Jean Piaget's Constructivism. Papert has described computers as cultural seeds that can be very powerful in influencing how people think. In particular three of the key concepts to Constructionism are presented: "objects to think with", public entities and microworlds.

Key words: CONSTRUCTIONISM/ EDUCATIONAL THEORYI COMPUTERS IN EDUCATION/ DIGITAL TECHNOLOGY/ EDUCATION/ PUBLIC ENTITIES/MICROWORLDS/ OBJECT-TO-THINK-WITH/

\section{Introducción}

¿QQué cosa fuera corazón, qué cosa fuera? ¿Qué cosa fuera la maza sin cantera? Un instrumento sin mejores pretensiones que lucecitas montadas para escena.

Silvio Rodríguez

La presencia de las tecnologías de la información y la comunicación (computadoras) en los diversos niveles y modalidades de la educación no solamente es una realidad bastante aceptada al iniciar el siglo XXI, sino que apunta a multiplicarse, y a llegar cada vez a

\footnotetext{
${ }^{1}$ Maestría en Educación con énfasis en Tecnología Educativa de la Universidad de Hartford, Connecticut. Profesora Asociada de la Universidad de Costa Rica desde hace 20 años donde imparte cursos de grado y posgrado y dirige investigaciones. También Investigadora Invitada en el Laboratorio de Medios del Instituto Tecnológico de Massachussets (MIT). Publica sistemáticamente en revistas especializadas y asiste con regularidad a eventos académicos internacionales. Domina los idiomas inglés y portugués. Más información en http://web.media.mit.edu/\%7Eeleonoral. Correo electrónico eleonora@media.mit.edu

2 Bachiller en Educación Preescolar por la Universidad de Costa Rica. Docente de educación preescolar y estudiante de la carrera Licenciatura en Educación Preescolar de la Universidad de Costa Rica. Actualmente desarrolla la investigación: Interacción: preescolares, bits y átomos. Correo electrónico alec 9cr@yahoo.com
}

Artículo recibido: 11 de junio, 2004

Aprobado: 28 de iunio. 2004 
mayores y más diversas poblaciones. La tendencia general ${ }^{3}$ es, sin embargo a que estas tecnologías sean incorporadas a los procesos de aprendizaje no como un medio para la construcción del conocimiento, sino como un fin en sí mismo. Es muy común observar cómo en un aula; en una institución educativa, o aún en un sistema educativo, la atención es acaparada por las máquinas en sí mismas (por la multiplicidad de sonidos, imágenes y movimientos que aportan y por la posibilidad de comunicación e interconexión). Pero es mucho menos frecuente (aunque hay algunas ${ }^{4}$ ) encontrar experiencias en las cuales los artefactos tecnológicos ocupan un claro lugar de herramienta, y en las que existe un verdadero fundamento pedagógico que oriente los procesos de aprendizaje. Lamentablemente, el "encandilamiento" y la emoción que provocan las tecnologías digitales cuando - parafraseando a Silvio Rodríguez ${ }^{5}$ - han sido incorporadas a los procesos de educación sin mayores pretensiones que lucirse en la escena, tiende a decaer de forma muy rápida. Es así como entonces las tecnologías digitales suelen sub y mal utilizarse en las aulas y en los sistemas educativos; el personal docente se siente amenazado por ellas; y los aprendices tienden a aburrirse, a aislarse o a desperdiciar el potencial de aprendizaje que guardan. Esta situación redunda, además, en una mala inversión de los (escasos) recursos que se asignan a la educación.

Si bien es cierto no hay una sola solución o receta suficiente para garantizar que las tecnologías digitales se usen en educación de manera que se apoyen el desarrollo y aprendizaje de los estudiantes, sí creemos que una adecuada fundamentación pedagógica que arroje luz sobre el potencial de estas tecnologías, es condición necesaria.

En los procesos educativos, es imprescindible tener clara la visión, las metas, las posibilidades y las limitaciones. El fundamento pedagógico (entre otros) colabora con la construcción de esa visión y con el establecimiento de expectativas.

Uno de los pensadores más reconocido internacionalmente por sus serias investigaciones en esta área es Seymour Papert del Laboratorio de Medios del Instituto Tecnológico de Massachussets MIT. Papert, además de crear herramientas digitales apropiadas para apoyar

${ }^{3}$ En Costa Rica y en muchos otros países en diversas latitudes del mundo.

${ }^{4}$ Como los Programas de Informática Educativa MEP-FOD; el Programa de Innovación Educativa del MEP o el Programa de Tecnologías Avanzadas de la Facultad de Educación de la UCR.

${ }^{5}$ Cantautor cubano de la nueva trova. 
el aprendizaje, propuso el Construccionismo como una teoría educativa que fundamente el uso de las tecnologías digitales en educación.

En este artículo hemos querido presentar la teoría construccionista de Papert, mirada desde una óptica pedagógica, haciendo énfasis en tres de los conceptos "papertianos" que consideramos más poderosos para la construcción de conocimientos utilizando tecnologías digitales: objetos para pensar (objects-to-think-with), entidades públicas y micromundos.

\section{Seymour Papert y Construccionismo: Generalidades}

Seymour Papert es un matemático que, a mediados del siglo pasado, observó la dificultad que presentan los niños y las niñas para operar las computadoras, a causa de que debían utilizar lenguajes de programación "serios" como Basic o Fortran, que les resultaban ininteligibles. Esta observación lo condujo a tomar dos decisiones importantes: estudiar profundamente con Jean Piaget su teoría epistemológica en Ginebra (entre 1958 y 1963) y asociarse con Marvin Minsky, el gran teórico de la inteligencia artificial, en Boston.

A partir de estas interacciones, Papert creó un lenguaje de cómputo con todas las potencialidades de los lenguajes "serios", pero con una sintaxis más análoga al lenguaje natural, más accesible para ser comprendido no solamente por los niños y las niñas, sino por jóvenes y adultos no expertos en computación. Se trata del lenguaje Logo, con el cual pueden operar las computadoras con mayor facilidad. Pero más aún, Papert influido por las ideas de Piaget, desarrolló un enfoque educativo para sustentar el uso de computadoras como herramientas de aprendizaje: el Construccionismo.

Además, junto a Marvin Minsky creó el Laboratorio de Inteligencia Artificial del Instituto Tecnológico de Massachussets MIT, donde construyeron un robot $^{6}$ que representaba una tortuga; éste se ponía en el piso y se conectaba a una computadora a través de la cual los aprendices programaban los movimientos del robot mediante el lenguaje Logo. De los movimientos de la tortuga surgieron las instrucciones básicas Logo (adelante, atrás, derecha

\footnotetext{
${ }^{6}$ Los robots nacen del área de la Inteligencia Artificial. Un robot es un sistema basado en estructuras mecánicas poliarticuladas, dotado de un cierto grado de "inteligencia" de manera que puede ser programado para que ejecute un comportamiento determinado. (concepto basado en definición de García, 2000)
} 
e izquierda) llamadas "primitivas". A partir de estas instrucciones primitivas y con las nociones básicas de la geometría euclidiana, niños, niñas, jóvenes y adultos no expertos en temas relacionados con computadoras podían programar a la tortuga para que realizara trayectorias complejas.

Ya para 1980 el robot era sustituido por un gráfico en la pantalla de la computadora; pero manteniendo vigentes los principios educativos del Construccionismo.

De tal forma ensayando una programación para comunicarse con la tortuga y hacerla moverse, errando y corrigiendo los errores ("ensayo-error") es posible aprender de manera intuitiva, geometría y matemáticas, y llegar a elaboraciones más complejas. Para Papert, este proceso de ensayar, errar y corregir el error (ensayo- error) conduce a las y los aprendices a crear y aprender. Él lo llama un proceso de depuración (corrección del error). Al respecto menciona que "...los errores nos benefician porque nos llevan a estudiar lo que sucedió, a comprender lo que anduvo mal y, a través de comprenderlo, a corregirlo" (Papert, 1987, Pág. 135-136).

En 1980 Papert publicó el libro Desafío a la Mente: Computadoras, Niños e Ideas Poderosas, que "revolucionó la concepción que se tenía sobre la relación que se establece entre las niñas y niños con las computadoras". (Revista Red Escolar, s.f.)

Una idea interesante de Papert en ese libro, es que él concibe a la computadora como una portadora de semillas culturales, cuyos productos cognitivos trascenderán la presencia de material concreto: "el trabajo con computadoras puede ejercer una poderosa influencia sobre la manera de pensar de la gente, yo he dirigido mi atención a explorar el modo de orientar esta influencia en direcciones positivas" (Papert, 1987, p. 43).

En el Construccionismo, Papert otorga a los y las aprendices un rol activo en su aprendizaje, colocándolos como diseñadores de sus propios proyectos y constructores de su propio aprendizaje. Se trata de facultar ("empower") a los y las estudiantes para que asuman ese papel activo. En contraposición a la instrucción asistida por computadora (CAI por sus siglas en inglés) que promueve que la computadora enseñe y programe al usuario, el Construccionismo propone que sea éste quien programe a la computadora, ya que al hacerlo adquiere “... un sentido de dominio sobre un elemento de la tecnología más moderna y 
poderosa y a la vez establece un íntimo contacto con algunas de las ideas más profundas de la ciencia, la matemática y el arte de construcción de modelos intelectuales" (Papert, 1987, p.17-18).

Para Papert, la sociedad y la cultura juegan un papel fundamental para facultar a los aprendices y revertir su papel tradicional de únicamente receptores pasivos de información. Tal como apuntan al respecto Maraschin y Nevado (1994) si se pretende que los estudiantes:

"... construyan su propio conocimiento la cultura debe ser la encargada de facilitar los recursos necesarios que den soporte a dicha construcción del aprendizaje, ya que esta no puede darse a partir de formulaciones abstractas o en ausencia de materiales que la faciliten. Lo anterior supone un reto para la sociedad, al ser esta la principal encargada de proveer los medios necesarios para crear un ambiente de aprendizaje apto, en el cual los estudiantes cuenten con material concreto el que puedan experimentar y realizar sus construcciones"

De esta manera, Papert nos advierte que no basta solamente con proponerle al estudiante que se haga cargo de su aprendizaje y que asuma un papel activo. La sociedad y la cultura tienen una gran responsabilidad, pues deben poner a disposición los recursos que necesita para ello. Por supuesto, Papert se refiere específicamente a computadoras.

Por otra parte, al proponer el Construccionismo, y particularmente al lenguaje Logo para apoyar el aprendizaje, Papert distingue entre dos clases de conocimiento: el matemático y el matético. Al referirse al conocimiento matemático expresa que: "...las tortugas son sólo un pequeño rincón de una gran materia matemática, la geometría de la Tortuga, un tipo de geometría fácilmente "aprehensible" y portadora efectiva de ideas matemáticas muy generales" (Papert, 1987, p. 82). En cuanto al matético, lo concibe como conocimiento sobre el aprendizaje y según él mismo señala "para resolver un problema busca algo similar que ya comprendas" (Papert, 1987, p. 83), sugiriendo así la utilización de los conocimientos previos en la resolución de conflictos actuales y con ellos la construcción de conocimientos nuevos. Menciona que la diferencia entre lo que se "puede" y lo que "no se puede" aprender, no depende del contenido sino de la relación del sujeto con este. 
La construcción del conocimiento, según Papert comprende, a su vez, dos tipos de construcción, la primera tiene lugar "en la cabeza" de las personas, frecuentemente ocurre de manera especialmente provechosa cuando el niño está conscientemente involucrado en una construcción de tipo más público, es decir, que puede ser mostrada, discutida, examinada, probada o admirada, desde un castillo de arena o una casa de Lego, hasta una página de web o un programa de computadora. (Fabel, s.f.)

Tal como menciona Papert, "...el mejor aprendizaje no vendrá de encontrar las mejores formas para que el profesor instruya, sino de darle al estudiante las mejores oportunidades para que construya". (Fabel, s.f.). Esta es la premisa que va a regir el proceso de aprendizaje desde el enfoque construccionista, el cual supone que existe una habilidad natural en las personas para aprender a través de la experiencia, y para crear estructuras mentales que organicen y sinteticen la información y las vivencias que adquiere en la vida cotidiana.

Por otra parte, en la teoría construccionista de Papert se encuentran implícitos, entre otros, tres conceptos que consideramos instrumentales para brindar a los y las estudiantes las mejores oportunidades de construcción: objetos con los cuales pensar, entidades públicas y micromundos.

\subsection{Objetos para pensar}

Papert parte de la hipótesis de que gran parte de lo que ahora consideramos demasiado "formal" o "demasiado matemático" será aprendido con la misma facilidad cuando los aprendices se desenvuelvan en el mundo computacionalmente rico del futuro. Es por ello que centra su interés en el proceso de invención de objetos para pensar, (objects-to-thinkwith) dentro de un nuevo tipo de ambiente de aprendizaje, el cual supone la interacción entre los niños, las niñas y las computadoras. De esta forma, afirma que "...podemos liberarnos de la tiranía de las consideraciones superficiales y pragmáticas que dictaron en el pasado respecto de qué conocimientos deberían ser aprendidos y a qué edad." (Papert, 1987, Pág. 69)

Lo que Papert denomina como un "objeto para pensar" es un objeto que pueda ser utilizado por un sujeto, para pensar sobre otras cosas, utilizando para ello su propia construcción de dicho objeto. Papert tienen un especial interés en el papel que juegan los objetos físicos en 
el desarrollo del pensamiento. Él dice que creamos nuestro entendimiento del mundo al crear artefactos, experimentar con ellos, modificarlos y ver cómo funcionan.

Objetos para pensar o con los cuales pensar, son artefactos cognitivos "... que proporcionan conexiones entre el conocimiento sensorial y el conocimiento abstracto, y entre el mundo individual y el mundo social." (Ostwald, 1996). Un ejemplo muy ilustrativo de lo que un "objeto para pensar" es para Papert, son los engranajes, que presenta en la introducción de su libro "Desafío a la Mente."

En esta introducción Papert describe cómo los engranajes lo fascinaron siendo niño y le proporcionaron una manera concreta de pensar sobre proporciones matemáticas abstractas. El autor Don Norman (en Ostwald, 1996), explica que hay dos modos para interactuar con artefactos coginitivos, que corresponden con los modos sensorial y abstracto de Papert: el de experiencia y el reflexivo. En el modo de experiencia, la información es percibida y manipulada sin mayor esfuerzo aparente o retraso. El modo reflexivo requiere un esfuerzo mental para pensar en contrastar los varios cursos de acción. En el modo de experiencia, los objetos y el conocimiento se dan por sentado, mientras que en el modo reflexivo, el mundo se encuentra disponible para ser inspeccionado. Los artefactos de experiencia nos permiten interactuar con el mundo. Nos proveen de información que nos permiten interpretar situaciones a través de nuestras percepciones. El peligro es que contienen información tácita que no siempre reconocemos. Los artefactos reflexivos son mucho más explícitos en el conocimiento que contienen.

Es una realidad que existen diversas opiniones tanto a favor como en contra de las nuevas tecnologías y la interacción de los niños con ellas. Sin embargo, cabe reconocer que un buen uso de las mismas, como "objetos para pensar", bajo condiciones apropiadas, puede llevar al sujeto al desarrollo de capacidades intelectuales, tales como la adquisición de capacidades de búsqueda y resolución de problemas, de capacidades de razonamiento y representación formal, desarrollo de modelos de conocimiento, pensamiento y aprendizaje, y mejora de estilos cognitivos y de aspectos sociales y afectivos (Rodríguez y Roselló, 1987).

En consonancia con Piaget, Papert considera que no se puede separar el proceso de aprendizaje de lo que se aprende, y lo hace explícito al escribir que: "La comprensión del aprendizaje debe ser genética. Debe referirse a la génesis del conocimiento. Lo que un 
individuo puede aprender, y cómo lo aprende, depende de los modelos con que cuenta. Esto plantea, a su vez, la cuestión de cómo los aprendió. De tal modo, las "leyes de aprendizaje" deben referirse al modo en que las estructuras intelectuales se desarrollan una a partir de la otra y cómo adquieren, en el proceso, forma tanto lógica como emocional" (Papert, 1987; p.13). En este sentido, los objetos para pensar no pueden separarse del proceso mismo del aprendizaje, ni del contenido aprendido. Los objetos para pensar (computadoras entre ellos) pasan a ser una parte inherente de la construcción del conocimiento.

\subsection{Entidades públicas}

Otro de los conceptos del Construccionismo son las entidades públicas. Papert señala que el aprendizaje tiende a ser más robusto y ocurre de manera especialmente provechosa cuando el aprendiz está conscientemente involucrado en una construcción de tipo más público, es decir, que puede ser mostrada, discutida, examinada, probada o admirada desde un castillo de arena o una casa de Lego, hasta el diseño de una página de web o un programa de computadora. (Fabel, s.f.)

Papert denomina a esta construcción una "entidad pública", ya que permite representar visual o audidtivamente ideas y conceptos para experimentar con ellos. El objeto creado, al ser compartido con los demás, se convierte en una organización pública a través de la cual el aprendizaje construccionista es poderosamente reforzado (Harel, citado por Yartó, 2001).

En este sentido, es importante insistir en que no es solamente el proceso de construcción lo que hace que el aprendizaje sea significativo para el aprendiz. Tanto el proceso de creación como el producto final, deben ser compartidos con otros para que verdaderamente el aprendizaje sea robusto. Esto se produce cuando se habla con otros o se explican o muestran diagramas o esquemas. .

\subsection{Micromundos ${ }^{7}$}

Inicialmente es necesario hacer notar que un micromundo constituye por sí mismo una entidad pública y que utiliza como herramientas para su construcción objetos para pensar. Papert (1987) considera que para aprender algo los aprendices necesitan como

${ }^{7}$ En este apartado presentaremos el concepto de micromundos propuesto por Papert. No confundir con la versión multimedial del lenguaje Logo, que lleva el nombre comercial de "Micromundos". 
prerrequisito, tener experiencias directas y físicas, obtener los medios para conceptualizar y capturar el mundo de este conocimiento, y encontrar maneras para facilitar la apropiación personal del conocimiento: es decir, crear un microcosmos, un lugar para esto.

Junto a Marvin Minsky, Papert desarrolló y acuñó el concepto de micromundo, "como un modelo para realizar representaciones de una realidad inmediata sobre un tema, que será refinado o pulido por los alumnos, iniciando con un punto de partida que les permita crear sus propias "extensiones" (Mardach, s.f.).

Literalmente, un micromundo es un minúsculo mundo, dentro del cual el aprendiz puede explorar alternativas, probar hipótesis y descubrir hechos que son verdad en relación con ese mundo. Difiere de una simulación en que el micromundo es un mundo real, y no una simulación de otro mundo.

Para McClintock y Turnes, un micromundo es "... un ambiente de aprendizaje en el cual los estudiantes manipulan y controlan varios parámetros para explorar sus relaciones. Los micromundos más complejos son expandibles, permitiendo al estudiante usar su creatividad para personalizar y ampliar el ambiente del micromundo" (en Mardach, s.f.). Es decir, los micromundos más complejos permiten a los estudiantes no solamente manipular sus relaciones, sino también modificar su estructura original.

Por su parte Sacristán (s.f.) incorpora un matiz informático al término y señala como "micromundos computacionales", aquellos "ambientes que pueden ser definidos como conjuntos de herramientas computacionales abiertas para que el estudiante pueda explorar y construir ideas y conceptos a través de actividades de programación."

Estas definiciones comparten tres ideas fundamentales: primero, un micromundo es un ambiente creado que representa una realidad; segundo, brinda herramientas para la exploración; y tercero, dicha exploración lleva a la construcción de conocimientos.

Siguiendo con Mardach (s.f.), éste describe el micromundo como una materialización de un modelo matemático pertenenciente a una realidad, el cual permite facilitar la comprensión y ejercitación de esa realidad. Además menciona que este modelo reúne la simulación y el 
juego, para reconciliar la teoría, la exploración y la práctica, comparando el micromundo con un laboratorio de estudio, y esto ayuda en la comprensión y creación de nuevos conceptos.

Según el autor citado, la construcción de micromundos debe cumplir los siguientes objetivos:

- Favorecer el aprendizaje significativo de los contenidos

- Ejercitar habilidades relacionadas con el tema

- Ejercitar el uso de los principios en los que se funda el pensamiento lógico.

- Desarrollar la creatividad a través de la construcción de aplicaciones.

- Implementar metodologías de tipo social. (Mardach, s.f.).

En la exploración de un micromundo los aprendices tienen la oportunidad: "experimentar, poner en práctica sus ideas, plantear y probar hipótesis a través del uso de un lenguaje y del trabajo en equipo como oportunidad de interacción que enriquece la experiencia educativa" (Mardach, s.f.). Por su parte el micromundo debe ofrecer al sujeto algunas características tales como el "despertar interés al usuario, darle nuevas oportunidades de acción y plantear varios niveles de profundidad que permitan al usuario irse adentrando y crear esquemas tan complejos como el usuario quiera." (Mardach, s.f.).

Mardach (s.f.) hace mención de tres principios de lo que llama "La filosofía del micromundo", estos son:

1. Principio de poder o dominio: se refiere al "poder hacer", lo cual lo impulsa hacia la resolución autónoma de los conflictos, para adquirir dominio de la situación mientras fortalece su visión de sí mismo.

2. Principio de resonancia cultural: se trata que el micromundo responda al modelo cultural propio.

3. Principio de continuidad cognoscitiva: sugiere la necesidad de respetar los tiempos evolutivos individuales, y que los conocimientos construidos se integren armónicamente a los anteriores.

En este sentido es importante anotar que para el Construccionismo, el mejor ambiente para aprender es un micromundo, que incluya objetos para pensar (entre ellos computadoras) y las entidades públicas de los aprendices, en proceso de construcción. 


\section{Conclusión}

El uso de las tecnologías digitales ser irá generalizando cada vez más en contextos educativos y de aprendizaje. Sin embargo, por más atractivas y novedosas que resulten las tecnologías en sí mismas, es muy importante tener siempre en mente que son solamente herramientas y que el verdadero valor está en el abordaje pedagógico en que se sustenten. En ese sentido, la teoría educativa de Seymour Papert, el Construccionismo es una propuesta sólida sobre la cual asentar un programa educativo que utilice tecnologías digitales. Partiendo de las ideas de Jean Piaget, el Construccionismo visualiza a los aprendices en papeles muy activos en la construcción de su propio conocimiento. Es decir, se busca que las y los estudiantes programen las computadoras y no a la inversa. En palabras de Papert, la idea es facultar, dar poder (empower), a los aprendices para que se hagan cargo de su propio proceso de construcción de conocimiento. Pero al mismo tiempo Papert advierte que no basta con la intención de trasladar el poder de aprendizaje a los aprendices. Ellos y ellas solamente lo podrán hacer si la sociedad y la cultura los proveen con las herramientas (objetos para pensar) y las condiciones (micromundos) adecuadas. Es en ese contexto que los y las estudiantes podrán construir entidades públicas y en el proceso, su propio conocimiento.

Esta propuesta educativa de Papert, no solamente ofrece un fundamento educativo para el uso de tecnologías digitales en educación. En sí mismo, el Construccionismo puede ser visto por el personal docente, como un objeto para pensar y re-pensar los procesos de aprendizaje, el sistema educativo y la propia práctica pedagógica. Por ejemplo, al analizar las propuestas del Construccionismo los y las educadoras pueden preguntarse específicamente, ¿cuáles son los objetos para pensar con que estamos facultando a los estudiantes para hacerse cargo de su propio aprendizaje? Y ¿cuáles son las características y las condiciones de los micromundos en los que aprenden? Y más aún, ¿cómo es que se promueve que los estudiantes hagan público el conocimiento construido? ¿a través de proyectos 0 de diseños? o ¿ solamente por medio de exámenes?

Y la sociedad en general debería preguntarse ¿cuán relevantes y útiles son las herramientas y los ambientes de aprendizaje con que cuentan las actuales generaciones para enfrentar los retos de la sociedad posmoderna? 


\section{REFERENCIAS}

Del constructivismo al construccionismo. (s.f.). Recuperado de http://academico.uno.mx/rnevarez/sistemaseducativos/CONSTRUCTIVISMO.html

Dewey, John. (1925). El niño y el programa escolar. Publicaciones de la Revista Pedagógica. Madrid, España.

Falbel, Aaron. (s.f.) Herramientas con qué construir (y pensar). Recuperado de http://www.viagenius.edu.pe/revista/pagina13.htm

García, Angélica. (2000). Robótica Homepage. Recuperado de http://www.uan.mx/ juan/robot.html

Harel, I. (1999). Clickerati kids. Who are they? Mamamedia. 21st Century Learning. Recuperado http://www.mamamedia.com/areas/grownups/new/21 learning/home alt.html

Maraschin y Nevado. (1994). Recuperado de www.academico.uno.mx

Mardach, Alexander. (s.f.). Micromundos Ensamble. Recuperado de http://www.redacademica.edu.co/export/REDACADEMICA/ddirectivos/proyectos peda gogicos/micromundos/El Proyecto Micromundos/Documentos/pdf/Ensamble.pdf

Méndez, Zaira. (1995). Aprendizaje y cognición. San José, Costa Rica: EUNED.

Oribe, Irigoyen. (s.f.) La era digital ¿Sobran los maestros?. Lectura corta suministrada en el curso FD-2046 Seminario: El docente en la era informática. II ciclo, 2003. Universidad de Costa Rica.

Ostwald, Jonathan. (1996). Knowledge Constriction in Software Development: The Evolving Artifact Approach. Recuperado de http://www.cs.colorado.edu/ ostwald/thesis/home.html

Papert, Seymour. (1987). Desafío de la mente. Buenos Aires, Argentina: Ediciones Galápago.

Papert, Seymour. (1995). La máquina de los niños. Argentina: Ediciones Paidos.

Papert, Seymour. Works by Papert. Recuperado de http://www.papert.org/works.html.

Revista Red Escolar. (s.f.) LOGO: Una posibilidad interesante para el trabajo en el aula. Recuperado de http://www.redesc.ilce.edu.mx/redescolar/Revista/01/articulos/09.html

Rodríguez-Roselló, L. (1987). Logo y currículum. En M. Aguirregabiria (Coord.). Tecnología y Educación (pp. 187-194). Madrid, España: Narcea.

Sacristán Rock, Ana Isabel. (s.f.) Temas de Investigación. Recuperado de http://www.matedu.cinvestav.mx/asacristan.html)

Thomas y Méndez. (1991). Psicología del niño y del aprendizaje. San José, Costa Rica: EUNED. 